\title{
Um Sistema de Apoio ao Ensino da Disciplina de Sistemas Hidráulicos Baseado na Metodologia de Resolução de Problemas
}

\author{
Eder Ivan de Araujo Crespo \\ João Carlos Gluz \\ Programa de Pós-Graduação em Computação Aplicada (PIPCA) - Unisinos - São \\ Leopoldo - RS - Brazil \\ araujo.ivan@hotmail.com,jcgluz@unisinos.br
}

\begin{abstract}
Simulators are commonly used to help the teaching of hydraulics courses. Main existing simulators in the market today are proprietary, closed source solutions, typically compatible with only one operational system. This work proposes an educational system intended to help the teaching of hydraulics courses, which is composed of a supporting tool to the teacher, where are created and corrected problems in hydraulics, together with a mobile application that communicates with teacher supporting tool and provides an environment where the student can solve these problems by a panel for the design of hydraulic circuits. The application implements the modeling of signal flow, which allows the simulation of the direction and sense of the fluid. Evaluation experiments showed the potential pedagogical use of this system to support the discipline of hydraulic systems as well as identified issues related to its usability.
\end{abstract}

Resumo. Simuladores são empregados no ensino da disciplina de sistemas hidráulicos em cursos de instituições de ensino tecnológico em todo país. Os principais simuladores existentes hoje no mercado são soluções proprietárias de código fonte fechado, em sua maioria compatíveis com apenas um sistema operacional. Esse trabalho propõe um sistema de apoio à disciplina de sistemas hidráulicos, baseado na metodologia de resolução de problemas, que é composto por uma ferramenta de apoio ao professor, onde são criados e corrigidos problemas de hidráulica, em conjunto com um aplicativo para dispositivos móveis, que comunica-se com a ferramenta de apoio ao professor e proporciona um ambiente onde o aluno pode resolver esses problemas, através de um painel de desenho de circuitos hidráulicos. O aplicativo implementa a modelagem de fluxo de sinais, o que possibilita a simulação da direção e do sentido do fluído. Por meio de experimentos de avaliações verificou-se o potencial uso pedagógico desse sistema no apoio à disciplina de sistemas hidráulicos, bem como questões que envolvem a sua usabilidade.

\section{Introdução}

O ensino tem vivenciado nos últimos anos o surgimento de novas modalidades de aprendizagem influenciadas pela evolução das Tecnologias de Informação e 
Comunicação. Dentre essas modalidades destaca-se a aprendizagem móvel que surgiu com o a disseminação dos dispositivos móveis nas escolas em todo o país. Essa modalidade, o uso de tecnologias de informação ou comunicação móveis e sem fio oferece suporte a processos de aprendizagem [Saccol et al. 2011] [Saccol et al., 2011].

A disciplina de sistemas hidráulicos é ministrada em cursos de nível técnico como mecânica industrial, automação e também em cursos de nível superior. Os softwares de desenho e simulação de circuitos hidráulicos são comumente utilizados nessa disciplina como ferramenta de apoio ao ensino para consolidar os conhecimentos dos aprendizes através da aprendizagem por descoberta. A aquisição desses softwares pelas instituições de ensino e até pelos alunos trazem importantes considerações no momento de sua escolha tais como, os custos de licenciamentos, as configurações necessárias para o ambiente de instalação (hardware e sistemas operacionais), entre outras. Após um levantamento dos principais softwares de simulação de circuitos hidráulicos existentes no mercado (ver seção 3), verificou-se uma série de restrições importantes referentes aos custos de licenciamento e disponibilidade de plataformas de hardware e software, incluindo mobilidade (suporte a dispositivos móveis). Em sua maioria, os simuladores são restritos a apenas um sistema operacional o que restringe a possibilidade de uso nas diferentes plataformas existentes atualmente.

O presente trabalho inspira-se na modalidade de aprendizagem móvel e no cenário atual dos softwares de simulação de circuitos hidráulicos para propor um sistema de apoio ao ensino da disciplina de sistemas hidráulicos baseado na metodologia de resolução de problemas. Conforme [Saccol et al. 2011], existe uma carência de metodologias e práticas pedagógicas desenvolvidas especificamente para um contexto de aprendizagem móvel, por se tratar de uma modalidade recente e que ainda não foi totalmente explorada pelos professores e pesquisadores. Assumindo a possibilidade de usar essa metodologia como uma opção relevante dentro do ensino da disciplina de sistemas hidráulicos, optou-se pelo seu emprego no desenvolvimento desse trabalho.

O sistema HyDrawing de apoio ao ensino da disciplina de sistemas hidráulicos proposto neste trabalho é um software aberto e livre, composto de uma ferramenta web de apoio ao professor, onde os problemas são criados e enviados aos dispositivos e onde posteriormente serão corrigidos, e por um aplicativo para tablets, onde aluno encontra um ambiente para a resolução de problemas que envolvam a construção de circuitos hidráulicos e sua validação através da simulação.

\section{Referencial Teórico}

A palavra grega hidráulica é formada pela união das palavras hidra que significa água e a palavra aulos que significa condução. A hidráulica é um campo da física que estuda o comportamento dos líquidos em movimento e em repouso, sendo responsável pelo estudo das leis que se aplicam ao transporte, conversão de energia, regulagem e controle de fluído, tendo impacto sobre suas variáveis (pressão, vazão, temperatura, viscosidade, etc.) [Fialho 2013]. Um sistema hidráulico tem como objetivo principal, converter uma forma de energia de entrada, no caso a energia dos fluídos (óleo hidráulico, água, etc.), em uma energia mecânica útil para realizar diversas tarefas como por exemplo movimentar o braço de uma escavadeira, subir e descer pontes e elevadores, rotacionar brocas, furadeiras, etc. Segundo Linsingen [2013; p. 17] "Um sistema hidráulico é um 
conjunto de elementos físicos convenientemente associados que, utilizando um fluído como meio de transferência de energia, permite a transmissão e controle de forças e movimentos".

\subsection{Simulação de Sistemas Hidráulicos}

A simulação é o estudo do comportamento de sistemas reais através do uso de modelos representativos desses sistemas, sendo que na simulação computacional verifica-se o uso de computadores no processo de simulação. Os programas de simulação possibilitam a apresentação de fenômenos, experiências e a vivência de situações difíceis, oferecendo cenários das mais variadas áreas de conhecimento, onde o usuário pode tomar decisões e obter de forma imediata as consequiências de suas escolhas [Moran et al., 2013].

Para [Chwif e Medina 2010], um modelo representa a abstração de uma realidade, a qual se aproxima do comportamento real de um sistema, mas com menor complexidade que o sistema verdadeiro. Em simulações computacionais, um modelo é um tipo particular de modelo matemático do sistema, que utiliza notações simbólicas e relações matemáticas para representar o sistema simulado. A modelagem por fluxo de sinal é uma abordagem tradicional usada na simulação, que se encontra atualmente bem desenvolvida e com seu uso difundido na modelagem de sistemas de controle [Silva e Nascimento 2014]. A abordagem consiste em representar os componentes do sistema por blocos que transferem um único valor para outro bloco sempre em uma única direção.

Assim como na modelagem de sistemas de controle, a modelagem do funcionamento dos componentes hidráulicos desse trabalho considera a transmissão de apenas uma variável entre os componentes, evitando assim um aumento na complexidade dos modelos. Dessa forma, através de álgebra booleana são definidas as equações que representam os modelos lógicos dos componentes. A figura 1 exibe um circuito hidráulico composto por um cilindro de ação dupla, uma válvula $5 / 2$ e uma válvula 4/2.

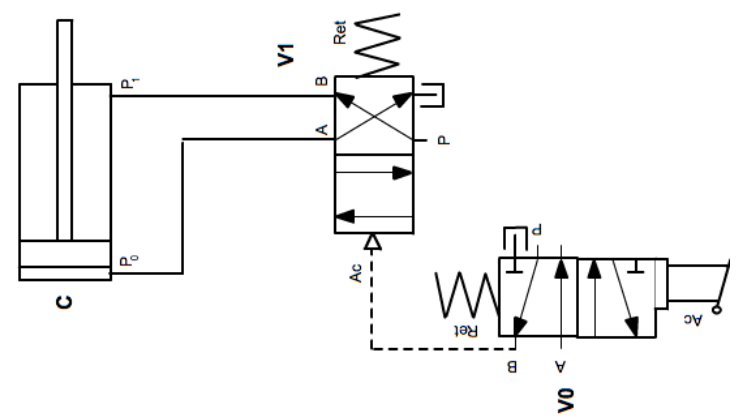

Figura 1 - Exemplo de sistema hidráulico

\subsection{Metodologia de Resolução de Problemas}

Um problema é uma situação a qual um indivíduo ou um grupo de indivíduos necessita solucionar, mas não possui um caminho rápido e direto que leve à sua solução, ou seja, quando não dispomos de algum processo automático que permita solucioná-la de uma forma mais ou menos imediata, de forma a descartar a necessidade de todo um processo de reflexão e de decisões a serem tomadas sobre passos que devem ser seguidos. Neste contexto, é possível diferenciar um problema de um exercício, já que nesse último, 
dispomos de mecanismos que nos levam a solução de maneira imediata. [Pozo 1998]. A metodologia de resolução de problemas é uma abordagem que parte do princípio de que o sujeito é um ser curioso, histórico e cultural, com capacidade para desenvolver a sua autonomia e a sua autoria. Essa metodologia é desenvolvida a partir da identificação e da resolução de problemas, onde a aprendizagem é motivada por meio de desafios, de situações-problema, geralmente baseadas em situações cotidianas, criadas por professores, estudantes ou pela colaboração destes [Saccol et al. 2011].

A metodologia de resolução de problemas encontra grande aplicabilidade no âmbito da disciplina de sistemas hidráulicos no que concerne o ensino de circuitos hidráulicos, sendo desenvolvida através da formulação de enunciados que podem envolver problemas nos mais diversos campos de aplicação da hidráulica, como por exemplo na indústria, nas aplicações navais, na engenharia civil, na mineração, na área hospitalar, no processamento de lixo urbano e também em nossas casas e edificações. Porém, o desenho e a interpretação de um circuito hidráulico demanda um conhecimento por parte do aluno, que é adquirido através de uma aprendizagem seqüencial passando pelo aprendizado dos diversos componentes como motores, cilindros e válvulas, e também na resolução dos problemas propostos em aula pelo professor, mediante o desenho e o estudo dos vários componentes de um circuito e as relações existentes entre eles.

\section{Trabalhos Relacionados}

Dois critérios definiram a busca por trabalhos relacionados: a identificação de aplicações de projeto e simulação de sistemas hidráulicos, aptas para uso em educação, e a identificação de trabalhos científicos visando o apoio ao ensino da disciplina de hidráulica visando. A tabela 1 apresenta um resumo do levantamento dos principais softwares de simulação existentes hoje no mercado.

Tabela 1 - Softwares de Desenho e Simulação de Circuitos Hidráulicos

\begin{tabular}{|c|c|c|c|}
\hline Nome & $\begin{array}{l}\text { Sistema } \\
\text { Operacional }\end{array}$ & Licenca & Desenvolvedor \\
\hline $\begin{array}{l}\text { V-HPS Hidraulic } \\
\text { and Pneumatic } \\
\quad \text { Simulator }\end{array}$ & Windows & $\begin{array}{c}\text { Paga, não possui } \\
\text { versão Trial }\end{array}$ & 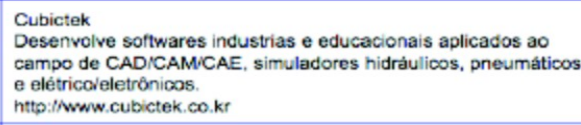 \\
\hline FluidSim & Windows & $\begin{array}{c}\text { Paga, possui } \\
\text { versão Trial }\end{array}$ & $\begin{array}{l}\text { Festo } \\
\text { Desenvolve equipamentos hidráulicos voltados à área } \\
\text { educacianal e componentes hidráulicos para a industria. } \\
\text { htto://ww/w.festo-didatic.com }\end{array}$ \\
\hline $\begin{array}{c}\text { Automation } \\
\text { Studio }\end{array}$ & Windows & $\begin{array}{l}\text { Paga, não possui } \\
\text { versão Trial }\end{array}$ & 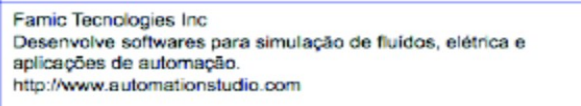 \\
\hline SimHydraulics & MacOs, Windows & $\begin{array}{c}\text { Paga, possui } \\
\text { versão Trial }\end{array}$ & $\begin{array}{l}\text { MathWorks } \\
\text { Lider no desenvolvirnento de softwares de computação } \\
\text { matemática. } \\
\text { htṭ:l/Awww.mathworks.com }\end{array}$ \\
\hline Hypneu & Windows & $\begin{array}{c}\text { Paga, possui } \\
\text { versão Trial }\end{array}$ & $\begin{array}{l}\text { Bardyne } \\
\text { Desenvolve o software HyPneu, publica livros na área de } \\
\text { engenharia de fluido e ministra cursos de treinamento } \\
\text { httto:l/wwww.hypheu.com }\end{array}$ \\
\hline $\begin{array}{c}\text { LVSIM Hyd \& } \\
\text { Pneu }\end{array}$ & Windows & $\begin{array}{c}\text { Paga, não possui } \\
\text { versão Trial }\end{array}$ & $\begin{array}{l}\text { Lab-Volt } \\
\text { Desenvolve sistemas de ensina e de tre inamento simulado para } \\
\text { cursos de engenharia. } \\
\text { hittp://wwww.labvolt.com }\end{array}$ \\
\hline
\end{tabular}


Na tabela 1 verifica-se que todos os softwares são soluções proprietárias com o código fonte fechado, o que inviabiliza a possibilidade de customizações para atender demandas específicas. Foram desenvolvidos para apenas para computadores de mesa ou notebooks, sem compatibilidade com tablets, trazendo desvantagens no aspecto mobilidade. Em sua maioria são suportados por apenas um sistema operacional o que restringe a possibilidade de uso nas diferentes plataformas de sistemas existentes.

A pesquisa bibliográfica foi empreendida sobre a base de trabalhos disponíveis nos eventos relacionados à Informática na Educação, em particular SBIE, WIE e LACLO, além de pesquisas sobre teses e dissertações disponíveis na Internet. No decorrer do levantamento bibliográfico foram encontrados poucos trabalhos que tratam sobre o desenvolvimento de aplicações de apoio ao ensino da disciplina de hidráulica. Entre eles, o de maior relevância para efeitos de comparação com esse trabalho foi a dissertação de mestrado de D. A. C. Pequeno [Pequeno 2004], cujo foco principal é o desenvolvimento de um software de propósito educacional e de uso presencial para simulação de circuitos pneumáticos, denominado Simula, com modelo matemático baseado na lógica fuzzy. Apesar do enfoque em sistemas pneumáticos, grande parte dos conceitos apresentados nesse trabalho podem ser aplicados à disciplina de hidráulica. A modelagem fuzzy foi aplicada na concepção do modelo matemático dos circuitos. utilizando como parâmetros de simulação as variáveis de potência hidráulica (pressão e vazão) e potência mecânica (força e velocidade). A modelagem por fluxo de sinal foi usada para especificar o funcionamento dos componentes, onde cada porta transmite uma única variável entre componentes.

Apesar do sistema proposto neste trabalho e do sistema Simula [Pequeno 2004] possuírem propósitos educacionais e disponibilizarem um ambiente para as tarefas de desenho e simulação de circuitos, observa-se as seguintes diferenças entre eles: a) $\mathrm{O}$ aplicativo proposto nesse trabalho tem como plataforma alvo os dispositivos móveis tablets, o que garante uma maior mobilidade do aluno em relação ao Simula. b) O Simula foi concebido para uso presencial. O aplicativo desse trabalho é concebido como uma ferramenta para ensino à distância, mantendo comunicação com a ferramenta de apoio ao professor, por meio do qual são criados novos problemas e corrigidas as soluções recebidas dos dispositivos. c) A simulação dos circuitos no presente trabalho é baseada em equações que definem a operação dos componentes, simulando a direção e o sentido que o fluxo toma durante a interação do usuário com as válvulas e demais componentes que controlam o fluxo do circuito. Apesar de ser possivelmente mais eficiente, por conta da menor quantidade de processamento necessária, essa implementação permite simulações menos complexas, se comparada à simulação baseada em lógica fuzzy empregada no Simula, que abrange outros comportamentos do circuito como velocidades de deslocamento de cilindros e forças aplicadas.

\section{Arquitetura do Protótipo}

Os problemas criados no sistema possuem um ciclo de vida que inicia-se na formulação realizada na ferramenta de apoio do professor, passando pela resolução em um aplicativo disponível em tablet, e finalizando com sua correção na ferramenta do professor. 
O ciclo de vida impacta diretamente nos requisitos de uso da aplicação. Para modelar esses requisitos foram criados casos de uso que envolvem os três tipos de atores do sistema HyDrawing: Aluno, Professor e Administrador.

A figura 2 ilustra os principais casos de uso do ator Aluno. Esses casos de uso são executados no aplicativo e estão ligados à resolução dos problemas propostos pelo ator Professor.

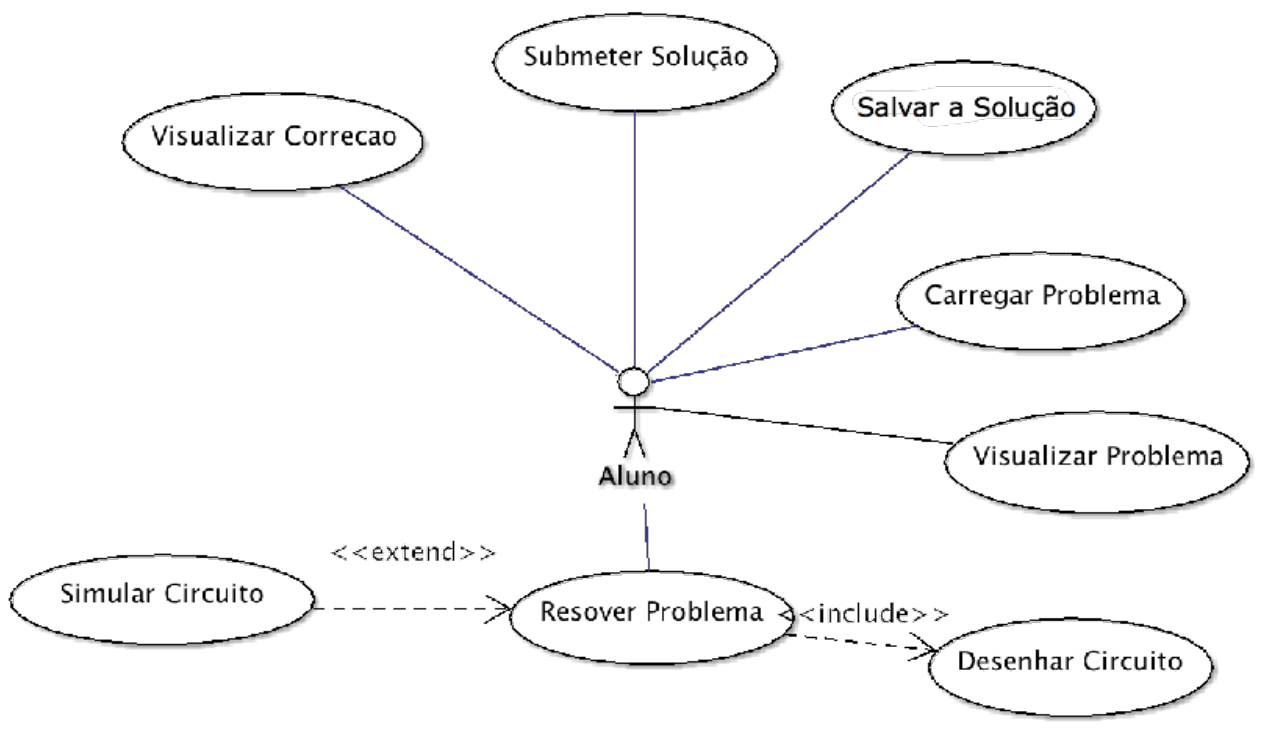

Figura 2 - Casos de Uso do Ator Aluno

O aplicativo do aluno é uma aplicação desenvolvida em HTML 5, cujas requisições que para a ferramenta de apoio ao professor são feitas por meio de tecnologia Ajax. A ferramenta do professor é um sistema web que mantêm um conjunto de servlets responsáveis por capturar essas requisições, executar o tratamento necessário, e devolver uma reposta para o aplicativo em um formato padrão JSON. A figura 3 ilustra a arquitetura do sistema proposto nesse trabalho.

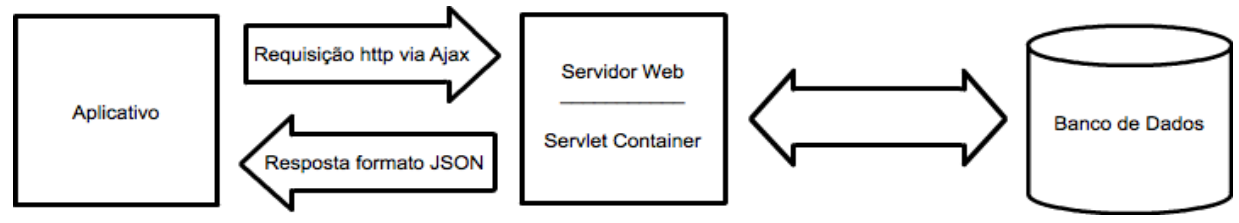

Figura 3 - Arquitetura Cliente Servidor do Sistema

O painel de desenho e simulação dos circuitos representa o ambiente de maior uso do aplicativo de apoio ao aluno. A figura 4 exibe a interface desse painel destacando suas funcionalidades.

\section{Metodologia de Avaliação}

A avaliação do aplicativo consiste em uma etapa fundamental desse trabalho, necessária para determinar a sua adequação à critérios ergonômicos e pedagógicos que devem estar presentes em softwares de propósito educacional. Para esta avaliação foi utilizada uma instrumento de avaliação denominado Técnica de Inspeção de Conformidade Ergonômica de Software Educacional (TICESE) [Gamez 1998]. Este instrumento está centrado na ergonomia de software aplicada a produtos educacionais informatizados, 
considerando tanto aspectos pedagógicos como aspectos que dizem respeito à interface dessa categoria de produto durante o processo de avaliação.

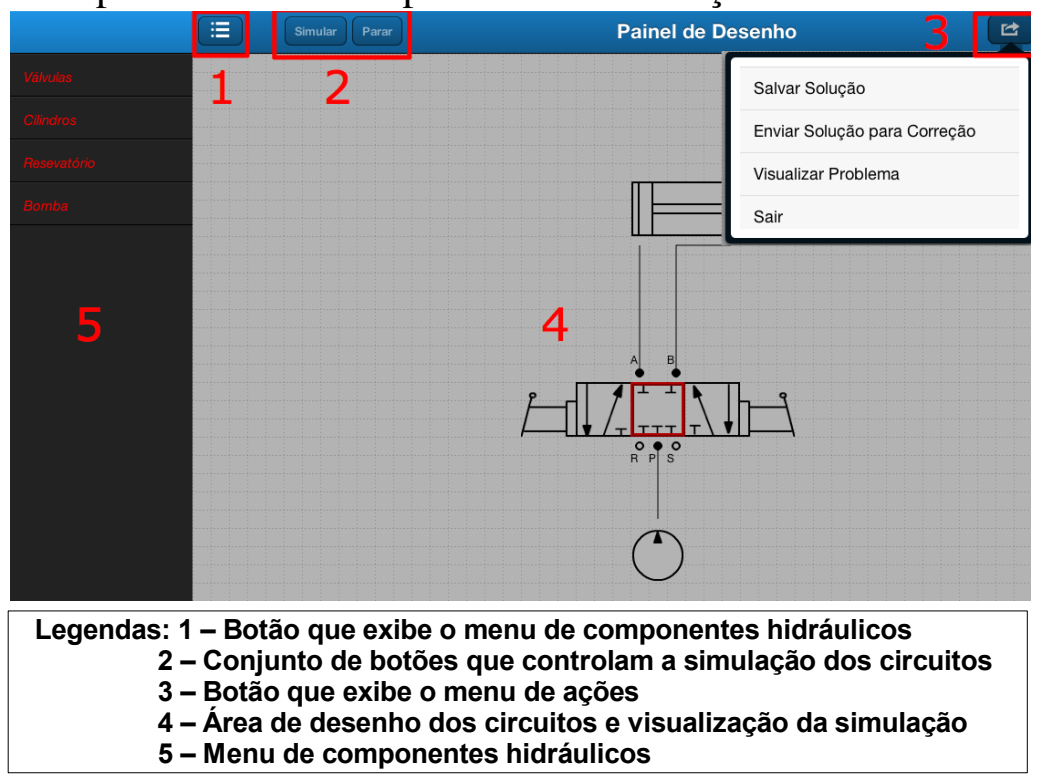

Figura 4 - Painel de Desenho dos Circuitos e seus Componentes

Para a aplicação dessa técnica é sugerida a formação de uma equipe multidisciplinar de avaliadores que possuam experiência no uso de produtos educacionais informatizados. A ferramenta também sugere um profissional com conhecimentos em Ergonomia de Interação Homem Computador, por ser uma técnica que considera questões de usabilidade de dispositivos interativos. A equipe de avaliação do aplicativo foi definida da seguinte forma: a) avaliador 1 - Mestre em Computação, leciona a disciplina de Interfaces Homem Computador (IHC) no curso de Ciências da Computação pela Universidade de Universidade de Santa Cruz do Sul (UNISC), tem 2 anos de experiência lecionando esta disciplina; b) avaliador 2 - Mestre em Ciências da Computação, leciona a disciplina de IHC no curso de Engenharia da Computação pela Universidade do Vale do Taquari (UNIVATES), tem 4 anos de experiência lecionando essa disciplina; c) avaliador 3 - Mestre em Sistemas e Processos Industrias, leciona a disciplina de Sistemas Hidráulicos e Pneumáticos do curso de Engenharia Mecânica da UNISC, tem 3 anos de experiência lecionando essa disciplina e 8 anos lecionando disciplinas relacionadas ao tema; d) avaliador 4 - Bacharel em Engenharia de Controle e Automação, leciona a disciplina de Automação Industrial do curso Técnico de Eletro Eletrônica da UNIVATES, possui 1 ano de experiência lecionando esta disciplina.

A TICESE adota uma categorização de softwares educativos baseada em atributos existentes no software. Dentre essas categorias, o aplicativo proposto enquadrou-se na categoria de Simulação e Modelagem, por ser uma ferramenta com foco no desenho e na simulação de circuitos hidráulicos. Considerando a categoria do aplicativo, foram selecionados 10 critérios definidos pela TICESE [Gamez 1998]: 1) Condução 2) Carga Informacional; 3) Concisão; 4) Ações Mínimas; 5) Densidade Informacional; 6) Consideração da Experiência do Utilizador; 7) Qualidade das Mensagens de Erro; 8) Proteção Contra Erros; 9) Avaliação do Aprendizado; 10) Adequabilidade.

O formulário de avaliação do aplicativo foi desenvolvido e disponibilizado a partir da ferramenta de apoio ao professor. Dentro do formulário foram descritos os 
conceitos de cada critério para facilitar o entendimento desses. Para os Avaliadores 1 e 4 o aplicativo e a ferramenta de apoio ao professor foram disponibilizados para a plataforma web. Os Avaliadores 2 e 3 realizaram a avaliação do aplicativo em um dispositivo da marca IPAD 2. Antes da avaliação ocorrer foram realizados encontros presencias com cada professor, onde foi exposto o trabalho e a metodologia de avaliação a ser aplicada.

Para contemplar todas as etapas do ciclo de vida do problema foi criado um cenário de uso do sistema a ser executado no decorrer da avaliação. Neste cenário, o avaliador no papel de professor cria um enunciado de problema sobre um sistema hidráulico simples e cadastra esse problema na ferramenta de apoio ao professor. $\mathrm{Na}$ continuação, o avaliador faz o papel do aluno e procede com a resolução deste problema na aplicação tablet destinada ao aluno. Nesta etapa do cenário, o avaliador acessa o tutorial e o workflow, seleciona o problema a ser resolvido, navega pelas opções da aplicação e execute operações incorretas e inválidas antes de finalizar a solução e encaminhá-la de volta ao professor. Após essas operações, o avaliador volta a ferramenta de apoio ao professor, procede com a análise e correção do problema recebido do aluno, retornando um feedback para o aluno através da ferramenta. Para finalizar, o avaliador volta ao papel de aluno, para verificar a recepção das correções e análises do professor na aplicação disponível no tablet.

\section{Resultados da Avaliação}

O cálculo do valor médio $\mathbf{X}(j)$ de cada critério $j$ foi realizado aplicando-se a seguinte equação [Gamez 1998]:

$$
\mathbf{X}(j)=\sum_{(i=1)}^{\mathrm{q}(j)} \frac{\sum \mathbf{a}(i) * \mathbf{p}(i)}{\sum \mathbf{p}(i) \mathrm{q}(j)} * 100
$$

onde, $\mathrm{q}(j)$ é número de questões por critério, $\mathbf{a}(i)$ é o valor da questão e $\mathbf{p}(i)$ o peso atribuído à questão. Essa equação foi aplicada à todos os critérios para observar o percentual de conformidade ergonômica do sistema em relação a cada critério avaliado.

As questões relacionadas aos critérios Avaliação do Aprendizado e Adequabilidade foram ignoradas pelos avaliadores 1 e 2, que não possuem experiência no ensino da disciplina de sistemas hidráulicos, evitando comprometer os resultados da avaliação pedagógica. A média dos critérios de Avaliação do Aprendizado e Adequabilidade para os avaliadores 3 e 4 foi, respectivamente, de $100 \%$ e $92,5 \%$. A figura 5 mostra um resumo com a média dos critérios ergonômicos analisados por todos os avaliadores.

Os critérios relacionados à ergonomia do aplicativo tiveram um percentual de inconformidade maior nas avaliações realizadas pelos avaliadores 1 e 2 em comparação com as avaliações realizadas pelo avaliadores 3 e 4 . Esse fato também pode ser atribuído à experiência dos avaliadores 1 e 2 na área de IHC, que trataram de forma mais rigorosa a avaliação. Neste contexto, o critério Condução demonstra um percentual de conformidade menor em todas as avaliações realizadas, com média de $71,14 \%$ e desvio padrão de $2,6 \%$, sendo um ponto crítico a ser melhorado no sistema. Dentre as inconformidades apresentadas pelos avaliadores, destacam-se algumas observações realizadas e que estão sendo objeto de revisão: “A fonte cinza sobre a cor branca do 
formulário de detalhamento do problema dificulta a sua leitura"; "Os títulos Tempo de Execução e Nota Estipulada do formulário do problema não são claros e objetivos.”; "Botão Simular não faz sentido caso ainda não haja um circuito construído."; "Se o circuito já foi enviado para correção, porque permitir a sua edição?".

A avaliações realizada pelos usuários 3 e 4 demonstraram um maior rigor nos aspectos funcionais do aplicativo e na verificação do uso das normas técnicas que estabelecem as regras na representação dos componentes. O critério pedagógico Adequabilidade avaliado pelo avaliadores 3 e 4 apresentou um percentual médio de conformidade de $92,5 \%$, com desvio padrão de $2,5 \%$ o que pode ser considerado um percentual alto de conformidade. As seguintes críticas e sugestões relatadas pelos avaliadores também estão sendo objeto de reconsideração: "Poderia ser melhor trabalhada questões dimensionais e de aplicação de normas (ISO e DIN) "; "Fazer uso de listas com limite de componentes disponíveis para um dado problema“; "Disponibilizar válvulas de segurança no menu de componentes."; "Válvulas com retorno por mola devem sempre retornar automaticamente"; "Disponibilizar blocos de construção prontos no menu de componentes“.

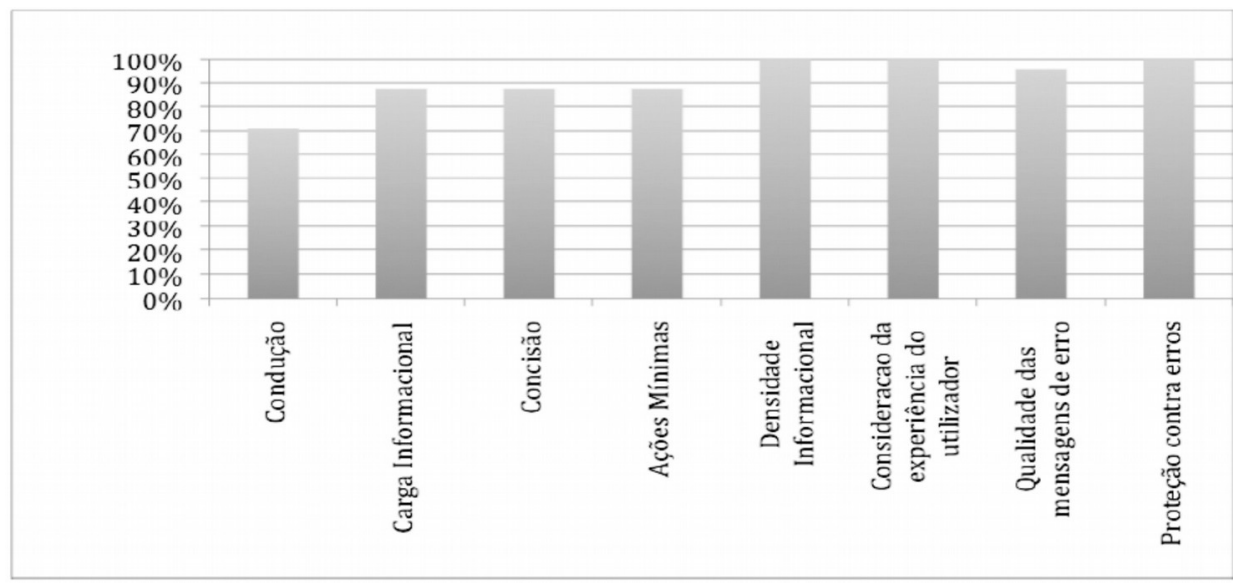

Figura 5. Média dos critérios ergonômicos de todos os avaliadores.

\section{Conclusões}

Os experimentos e avaliações fornecem evidências significativas que o sistema de ensino HyDrawing proposto neste trabalho mostra um grande potencial de uma nova ferramenta de apoio ao ensino da disciplina de hidráulica, que pode ser adotada em cursos de nível técnico e superior nas modalidade de ensino à distância, como também em cursos presenciais. Na simulação dos circuitos hidráulicos do aplicativo destinado ao aluno, foi utilizada a modelagem por fluxo de sinais, o que possibilita a verificação do sentido e da direção do fluxo do óleo nos circuitos. Este tipo de simulação facilita o entendimento das funções dos componentes hidráulicos e suas relações.

A ferramenta de apoio ao professor também é uma contribuição do presente trabalho, constituindo um ambiente que possibilita a criação e correção dos problemas e também o envio de feedbacks aos alunos.

As tecnologias utilizadas no desenvolvimento do sistema, foram selecionadas considerando a sua portabilidade para os diferentes dispositivos móveis, e também para a web, de maneira a não tornar o seu uso restrito ou dependente de alguma plataforma. 
A avaliação do sistema constitui-se em uma etapa muito importante de todo o trabalho. A aplicação da ferramenta TICESE mostrou que há muitas melhorias a serem realizadas no aplicativo considerando o critério de Condução. Nos demais critérios, o aplicativo demonstrou um alto percentual de conformidade.

Foram muitas as sugestões de melhorias colocadas pelos avaliadores. Outras idéias também surgiram no decorrer do desenvolvimento do sistema. Destaca-se as mais relevantes. Parâmetros como pressão, vazão, força e velocidade são simulados pelos softwares de simulação de sistemas hidráulicos comerciais. Assim, pode se pensar em uma modelagem desses parâmetros no aplicativo, o que enriqueceria o aprendizado do aluno com a possibilidade de simular diferentes comportamentos do circuitos.

Existem diversos mecanismos de avaliação da aprendizagem em ferramentas de ensino à distância. Uma sugestão de melhoria estaria na criação de alguns desses mecanismos na ferramenta do professor. Um exemplo seria armazenar as interações do aluno com o aplicativo no decorrer da solução de um problema proposto. Essas interações poderiam servir como base para verificar por exemplo, a quantidade de erros cometidos na criação de um circuito, ou outras estatísticas que favorecessem uma análise detalhada do comportamento do aluno e que auxiliasse o professor na melhoria de suas estratégias de ensino.

Considerando o critério de experiência do utilizador, poderia ser criado um tutorial com exercícios guiados, o qual poderia ser disponibilizado em um formato interativo, que facilitaria a aprendizagem dos conceitos mais básicos de hidráulica por alunos que estão iniciando a disciplina ou por pessoas com interesse em adquirir novos conhecimentos.

\section{Referências Bibliográficas}

Chwif, L. e Medina, A. C. (2010) Modelagem e Simulação de Eventos Discretos: Teoria e Aplicações. 3. ed, Ed. do Autor, São Paulo.

Fialho, A. B. (2013) Automação Hidráulica : Projetos, Dimensionamento e Análise de Circuitos. 6. ed, Ed. Érica LTDA, São Paulo.

Gamez, L. (1998) TICESE - Técnica de Inspeção de Conformidade Ergonômica de Software Educacional (Dissertação Mestrado em Engenharia Humana), Curso de Pós-Graduação em Engenharia Humana - Universidade do Minho, Portugal. 267f.

Linsingen, I. V. (2013) Fundamentos de Sistemas Hidráulicos, Ed. da UFSC, Florianópolis.

Pequeno, D. A. C. (2004) Simula - Um software educativo de simulação de circuitos pneumáticos com aplicações de lógica fuzzy (Dissertação Mestrado em Informática Educativa), Curso de Mestrado Integrado Profissionalizante em Computação Universidade Estadual do Ceará, Fortaleza. 141f.

Pozo, J. I. (1998) A solução de problemas: Aprender a resolver, resolver para aprender, Ed. Art. Med. Sul, Porto Alegre.

Saccol, A., Schlemmer, E. e Barbosa, J. (2011) M-Learning e U-Learning: Novas perspectivas da aprendizagem móvel e ubíqua, Prentice Hall, São Paulo.

Silva, J. C. e Nascimento, J. C. (2014) "Modelagem e Simulação Dinâmica : AMESim Ambiente para Protótipos Virtuais". http://nupet.daelt.ct.utfpr.edu.br/_ontomos/ paginas/Artigos/artigotecnico.pdf 